\title{
Tox-Zentrum: Brennpunkte 2002
}

\author{
M. Guirguis, C. Rauber-Lüthy, G. Egli, H. Kupferschmidt
}

Im Jahr 2002 beantwortete das Tox-Zentrum 33111 Anfragen zu Intoxikationen. Die Inanspruchnahme der Vergiftungsberatung stieg damit gegenüber den Vorjahren weiter an. Der Zugang zu diesem landesweiten Notfalldienst wird verbessert: Ab November 2003 ist das Tox-Zentrum zusätzlich über die nationale dreistellige Notfallnummer 145 erreichbar.

Aus dem reichen Erfahrungsfundus gut dokumentierter ärztlicher Verlaufsberichte kristallisierten sich im Berichtsjahr drei Schwerpunkte heraus: die Bedeutung eines national tätigen Notfallberatungsdienstes in der Toxikovigilanz, die nicht zu unterschätzende Toxizität von Naturtoxinen wie der Herbstzeitlose sowie die andauernde Problematik schwerer Intoxikationen mit klassischen trizyklischen Antidepressiva.

\section{Einleitung}

Die Anfragen an das Schweizerische Toxikologische Informationszentrum (Tox) haben im Jahr 2002 erneut zugenommen. Mit 33111 Beratungen stieg die Anzahl Anfragen innerhalb von fünf Jahren um insgesamt $12 \%$. Dem gesteigerten Informationsbedürfnis der Laien stand ein noch stärkerer Anstieg der ärztlichen Anfragen gegenüber, nahmen doch die Beratungen von Ärztinnen und Ärzten in der gleichen Zeitperiode um $14 \%$ auf 9878 zu. Bei 3041 ärztlichen Anfragen konnte eine Gefährdung der Patienten bereits zum Zeitpunkt des Anrufes ausgeschlossen werden. In dieser Situation wurde auf einen schriftlichen Follow-up verzichtet. Von den restlichen 6837 Fällen konnten 4161 durch den behandelnden Arzt gut dokumentierte Fälle detailliert analysiert werden. Erfreulicherweise blieben 852 Betroffene (20,5\%) symptomlos und 2357 Patienten (56,6\%) zeigten einen leichten Vergiftungsverlauf. Mittelschwere Intoxikationen wurden $639 \mathrm{mal}(15,4 \%)$ und schwere Vergiftungen $297 \mathrm{mal}(7,1 \%)$ registriert. 16 Intoxikationen $(0,4 \%)$ verliefen letal. Die Tabelle 1 zeigt die Verteilung der ausgewerteten Fälle bezüglich Schweregrad und Altersgruppe der betroffenen Patienten. Bei den Noxen standen die Medikamente mit $67 \%$ aller ärztlichen Anfragen deutlich im Vordergrund. In knapp $10 \%$ informierten sich die Ärzte zu Intoxikationen mit Haushaltsprodukten. Weitere häufige Noxengruppen waren technische und gewerbliche Produkte $(7 \%)$, Genussmittel wie Drogen und Alkohol (6\%) und Pflanzen (3\%).
Von den insgesamt 7817 verschiedenen Noxen oder Noxengruppen, die letztes Jahr beim Tox zu einer Anfrage führten, sind deren drei aus ganz unterschiedlichen Gründen bemerkenswert: Atembeschwerden durch Textilimprägnierungsspray, Vergiftungen durch Herbstzeitlosen, und Intoxikationen mit trizyklischen Antidepressiva.

\section{Toxikovigilanz: Epidemische Atem- wegsbeschwerden durch Textilimprägnierungssprays}

Textil- und Lederimprägnierungssprays führten in der Vergangenheit nur zu gelegentlichen Anfragen an das Tox (5- bis 10mal jährlich) und verursachten kaum je schwerere Symptome. Im letzten Quartal 2002 und Anfang 2003 kam es jedoch zu einer markanten Häufung von Fällen mit teilweise schweren Atemwegssymptomen nach Anwendung solcher Sprays, mit Husten, Dyspnoe, Lungenödem und toxischer Alveolitis (Abb. 1).

Die Fälle traten ausser in der Schweiz nur noch in Holland auf und waren mit wenigen bestimmten Produkten assoziiert, bei denen kurz vorher Änderungen an der Zusammensetzung vorgenommen worden waren. In Verbindung mit der erhöhten Toxizität gebracht wurden der Imprägnierstoff, ein Fluorocarbonpolymer, in Kombination mit einem neuen Lösungsmittelgemisch. Die genauen Ursachen werden derzeit untersucht. Es wird aber vermutet, dass die veränderte Formulierung einen feineren Sprühnebel erzeugt, der tiefer in die Atemwege gelangen kann. Der bessere Geruch der neuen Produkte hat zudem vielleicht zu einer sorgloseren Anwendung verleitet (grössere Mengen, Anwendung in geschlossenen Räumen).

Die Pneumotoxizität gewisser Fluorcarbonverbindungen ist gut bekannt. Bereits in den 80er und 90er Jahren sind in Deutschland und in den USA derartige Epidemien aufgetreten $[1,2]$. Möglicherweise verwandt mit diesem Problem ist das sogenannte Polymerrauchfieber, das durch Pyrolyse von zu stark erhitztem Teflon (Polytetrafluroethylen) zustande kommt. Es werden auch Fälle berichtet, wo es nach dem 
Abbildung 1

Schweregrad des Verlaufs nach Inhalation von Imprägnierungssprays.

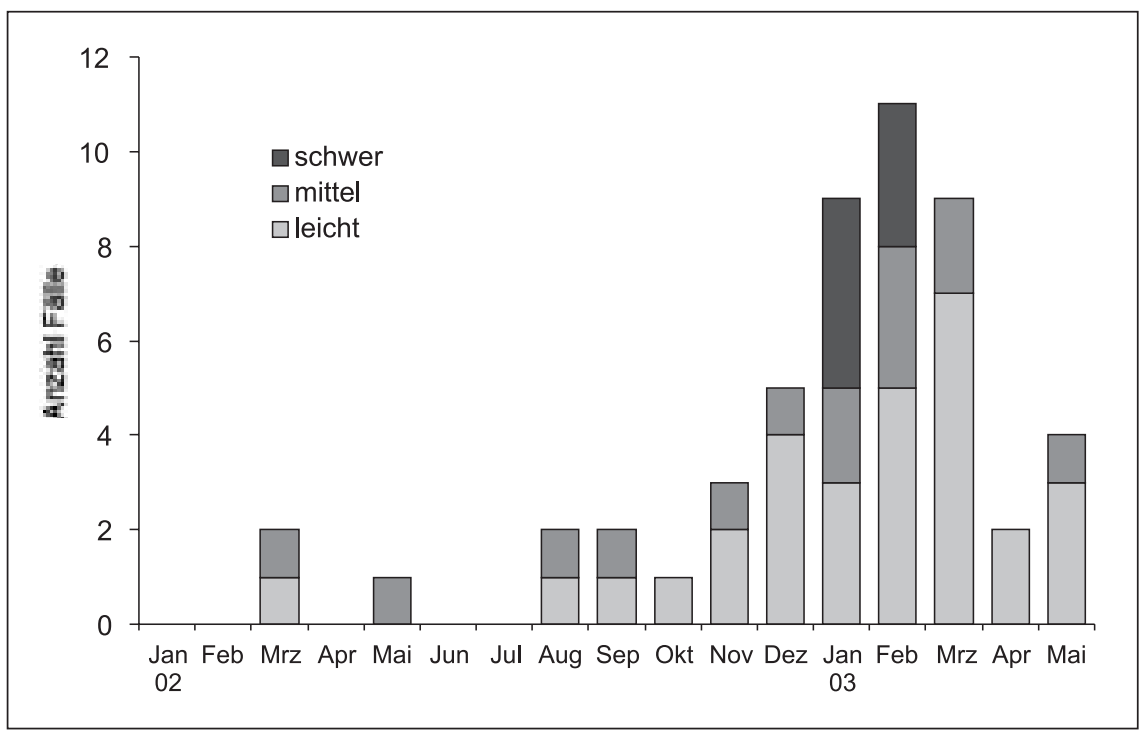

Rauchen von mit Fluorcarbonverbindungen kontaminierten Zigaretten zu vergleichbaren Symptomen kam [3, 4].

Die beteiligten Produkte wurden im Februar 2003 vom Markt zurückgezogen und die Bevölkerung umgehend vom Bundesamt für Gesundheit (BAG) und den Herstellern und Verkäufern der Sprays informiert. Diese Massnahmen führten - zusammen mit dem Einzug des Frühlings zum raschen Rückgang solcher Fälle (Abb. 1).

Die Produktsicherheit in der Schweiz ist hoch. Dennoch können vermeintlich unwesentliche Veränderungen der Produktzusammensetzung zu einer unerwarteten Steigerung der Toxizität führen. Die kontinuierliche aufmerksame und fachkundige Beobachtung der Vergiftungsaktivität (Toxikovigilanz) durch das ToxZentrum kann solche Zwischenfälle rechtzeitig erfassen und dient damit dem Schutz der Bevölkerung.

\section{Colchicin}

Im letzten Jahr verzeichnete das Tox drei tödliche Colchicinvergiftungen, die alle durch die Einnahme von Blättern der Herbstzeitlose zustande kamen. In zwei Fällen, die Erwachsene betrafen, handelte es sich um eine Verwechslung mit Bärlauch. Im dritten Fall war ein Kind betroffen, bei dem eine akzidentelle, nicht rechtzeitig erkannte Einnahme der Pflanze zu einem tödlichen Verlauf führte.

Colchicin ist das Gift der Herbstzeitlose (Colchicum autumnale), deren Blätter mit denen des
Bärlauchs verwechselt werden können. Dies geschieht allerdings nur im Frühling, wenn sowohl bei Bärlauch als auch Herbstzeitlose nur die Blätter $z u$ sehen sind. Im Herbst dagegen ist die Herbstzeitlose aufgrund der charakteristischen lilafarbenen Blüten für alle erkennbar. Aber nicht nur die Herbstzeitlose, sondern auch die Ruhmesblume (Gloriosa sp.) enthält das hochtoxische Colchicin. Vergiftungen mit dieser Pflanze sind aber extrem selten, da sie in der Schweiz nicht heimisch ist und auch keine Verwechslungsmöglichkeit mit einer essbaren Pflanze besteht. Eine andere Quelle für eine Colchicinintoxikation sind Medikamente mit diesem Wirkstoff, der vor allem beim Familiären Mittelmeerfieber, aber auch zur Schmerzbekämpfung beim akuten Gichtanfall eingesetzt wird. Colchicin wird in der Leber über das Cytochrom P450 3A4 metabolisiert. Da die therapeutische Breite des Colchicins sehr klein ist, sind Interaktionen mit Medikamenten oder Nahrungsmitteln zu erwarten, die dieses Enzym hemmen (Erythromycin, Ketoconazol, Grapefruitsaft).

Die Toxizität des Colchicins beruht auf einer Störung der Mikrotubuli in den Zellen. Dadurch kommt es zum Beispiel zur Hemmung der Zellteilung (Mitose). Dieses «Spindelgift» bindet intrazellulär reversibel an Tubuline, verhindert deren Polymerisation und dadurch den Aufbau der Mikrotubuli. Davon sind verschiedene Organsysteme betroffen, die Intoxikation verläuft klassischerweise in drei Phasen. Magendarmsymptome beginnen innert weniger Stunden. Die Volumendepletion im Rahmen der schweren Gastroenteritis kann zu schweren Elektrolytstörungen und zu hypovolämischem Schock führen. In der zweiten Phase nach ein bis drei Tagen kommt es zum Multiorganversagen, wobei in der Regel initial die Kardiotoxizität und später die Knochenmarksdepression im Vordergrund stehen. In der dritten Phase, die 7-10 Tage nach Einnahme beginnt, erfolgt die Erholung mit einer «Rebound»-Leukozytose, reversibler Alopezie und neuromuskulären Störungen. Letztere können noch Wochen andauern.

Eine kausale Therapie existiert nicht, und die Wirksamkeit der supportiven Behandlung ist beschränkt. Die wichtigste Massnahme ist eine sofortige primäre Dekontamination. Dazu kann die Magenspülung und/oder die Verabreichung von Kohle (1-2 g/kg Körpergewicht) eingesetzt werden. Diese Massnahme muss, vor allem nach der Einnahme von Tabletten, in der ersten Stunde erfolgen, da Colchicin rasch resorbiert wird. Bei Tabletten werden maximale Plasmaspiegel nach 30 bis 120 Minuten erreicht. Etwas protrahierter dürfte die Resorption des Colchi- 
cins aus der Pflanze erfolgen, was eine primäre Dekontamination auch noch über 1 Stunde nach Ingestion sinnvoll macht; trotzdem darf auch hier keine Zeit verlorengehen. Extrakorporelle Eliminationsverfahren sind wegen des grossen Verteilungsvolumens von Colchicin zuwenig effektiv. 1995 wurde der Fall einer Patientin aus Frankreich publiziert, die unter einer experimentellen Therapie mit Immunglobulin-FabFragmenten eine potentiell tödliche Colchicinvergiftung überlebte [5]. Hoffnungen auf eine vielversprechende Therapie sind aber verfrüht, da das Medikament heute nicht zur Verfügung steht.

\section{Trizyklische Antidepressiva}

Intoxikationen mit Antidepressiva sind relativ häufig. In 483 (11,6\%) der 4161 ausgewerteten Fällen aus der Jahresstatistik 2002 war ein Antidepressivum die toxikologisch relevante Noxe. Seit der Einführung der klassischen Trizyklika (TCA) in den 60er Jahren führten diese Medikamente auf Grund ihrer kardialen und zentralnervösen Toxizität regelmässig zu schweren und tödlichen Vergiftungen.

Mit dem Aufkommen der neuen Antidepressiva aus der Gruppe der Selektiven Serotonin- wiederaufnahmehemmer (SSRI) verband sich darum die Hoffnung auf einen Rückgang der schweren Vergiftungen, nachdem die in der Literatur publizierten und dem Tox gemeldeten Intoxikationen mit SSRI [6] einen wesentlich benigneren Verlauf zeigten als die klassischen TCAVergiftungen. Weil die trizyklischen Antidepressiva immer noch häufig eingesetzt werden, ist die Kenntnis über Symptomatologie und Therapie dieser potentiell lebensbedrohlichen Intoxikationen nach wie vor unerlässlich.

Da die klassischen Trizyklika als Antidepressiva oder mit anderen Indikationen (adjuvante Schmerztherapie, als Sedativa) weiter häufig verschrieben werden, hat sich diese Erwartung nicht erfüllt. Die häufigsten Vergiftungen mit einem Antidepressivum waren auch 2002 diejenigen mit Trimipramin. Vergiftungen mit Amitriptylin und Trimipramin sind etwa gleich häufig wie diejenigen mit den vier gängigsten SSRI Citalopram, Paroxetin, Sertralin und Fluoxetin, verlaufen jedoch wesentlich häufiger schwer (Abb. 2). Die beiden am häufigsten beteiligten TCA Amitriptylin und Trimipramin führten in $16 \%$ bzw. in $21 \%$ zu einem mittelschweren und in $33 \%$ bzw. $16 \%$ zu einem schweren Verlauf, und auch der einzige Todesfall war auf Trimipramin zurückzuführen. Im Gegensatz dazu verliefen Intoxikationen mit den genannten SSRI nur

Tabelle 1

Noxengruppen und Schweregrad des Verlaufs bei Kindern und Erwachsenen.

\begin{tabular}{|c|c|c|c|c|c|c|c|c|c|c|c|}
\hline \multirow{2}{*}{$\begin{array}{l}\text { Noxengruppen / } \\
\text { Schweregrad }\end{array}$} & \multicolumn{5}{|c|}{ Erwachsene } & \multicolumn{5}{|c|}{ Kinder } & \multirow[t]{2}{*}{ Total } \\
\hline & 0 & $\mathbf{L}$ & M & $s$ & $\mathbf{T}$ & 0 & L & M & $S$ & $\mathrm{~T}$ & \\
\hline Medikamente & 296 & 1380 & 364 & 204 & 7 & 279 & 219 & 43 & 13 & - & $2805(67,4 \%)$ \\
\hline Haushaltsprodukte & 29 & 120 & 24 & 7 & 1 & 92 & 118 & 8 & 1 & 2 & $402(9,7 \%)$ \\
\hline $\begin{array}{l}\text { Technische und } \\
\text { gewerbliche Produkte }\end{array}$ & 24 & 170 & 45 & 15 & - & 17 & 27 & 5 & 2 & - & $305(7,3 \%)$ \\
\hline $\begin{array}{l}\text { Genussmittel, Drogen } \\
\text { und Alkohol }\end{array}$ & 12 & 91 & 86 & 32 & 2 & 12 & 17 & 3 & 1 & - & $256(6,1 \%)$ \\
\hline Pflanzen & 9 & 25 & 7 & 1 & 2 & 34 & 21 & 2 & - & 1 & $102(2,5 \%)$ \\
\hline (Gift-)Tiere & 1 & 25 & 8 & 3 & - & 2 & 11 & 1 & - & - & $51(1,2 \%)$ \\
\hline Pilze & 2 & 12 & 19 & 2 & 1 & 5 & 4 & 1 & - & - & $46(1,1 \%)$ \\
\hline $\begin{array}{l}\text { Körperpflegemittel } \\
\text { und Kosmetika }\end{array}$ & 5 & 11 & 1 & 1 & - & 7 & 17 & 2 & - & - & $44(1,0 \%)$ \\
\hline $\begin{array}{l}\text { Produkte für } \\
\text { Landwirtschaft }\end{array}$ & 6 & 16 & 2 & 4 & - & 9 & 3 & 3 & - & - & $43(1,0 \%)$ \\
\hline $\begin{array}{l}\text { Nahrungsmittel } \\
\text { und Getränke }\end{array}$ & - & 13 & 5 & 2 & - & 2 & 1 & 1 & - & - & $24(0,6 \%)$ \\
\hline Veterinärarzneimittel & 1 & 4 & 1 & - & - & 1 & 2 & - & - & - & $9(0,2 \%)$ \\
\hline $\begin{array}{l}\text { Andere oder } \\
\text { unbekannte Noxen }\end{array}$ & 2 & 43 & 7 & 6 & - & 5 & 7 & 1 & 3 & - & $74(1,9 \%)$ \\
\hline Total & 387 & 1910 & 569 & 277 & 13 & 465 & 447 & 70 & 20 & 3 & $4161(100 \%)$ \\
\hline
\end{tabular}

Schweregrad des Verlaufs: $\mathrm{O}=$ asymptomatisch; $\mathrm{L}=$ leicht; $\mathrm{M}=$ mittel; $\mathrm{S}=$ schwer; $\mathrm{T}=$ tödlich. 
Abbildung 2

Intoxikationen mit Trizyklischen Antidepressiva und Selektiven Serotininwiederaufnahmehemmern im Vergleich.

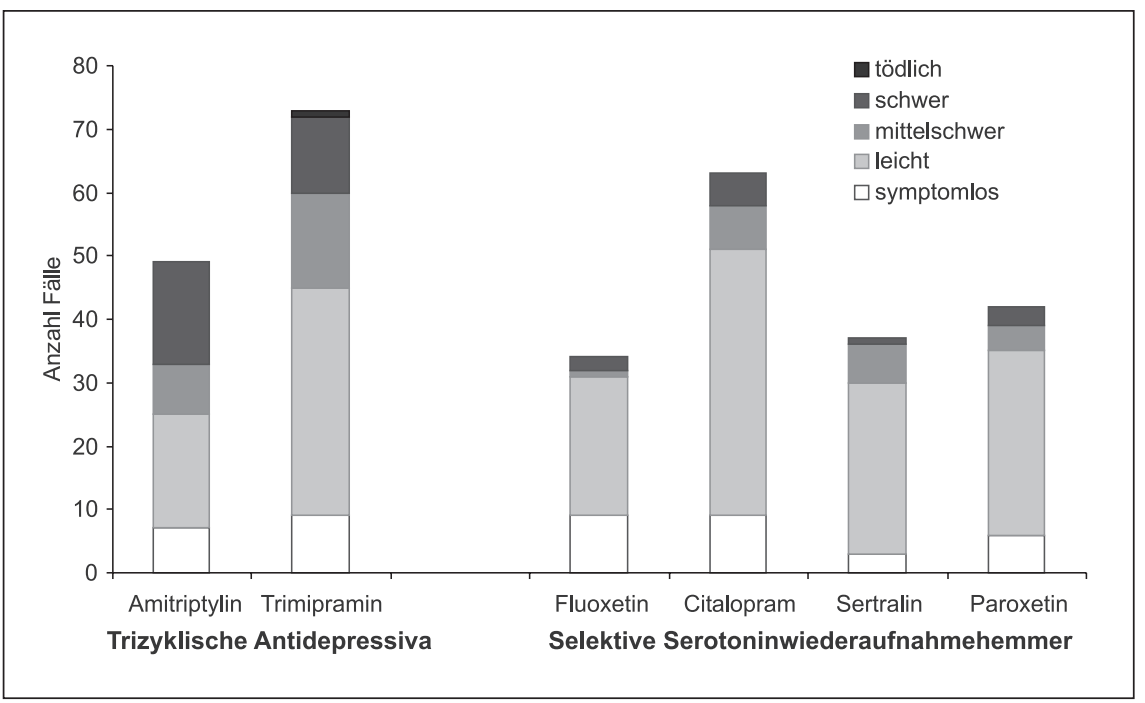

in 3 bis $8 \%$ schwer. Todesfälle waren mit einem SSRI nicht zu verzeichnen. Trizyklika führen nach Überdosierung zu einem anticholinergen Syndrom mit Koma oder zentralnervöser Erregung, erhöhtem Muskeltonus, Halluzinationen und Krampfanfällen. Die gefürchteten kardiovaskulären Störungen sind Tachykardie, AVBlockierungen, Bradykardie, ventrikuläre und supraventrikuläre Arrhythmien, arterielle Hypotonie, Schock und Herzstillstand. Sie kündigen sich im EKG durch eine Verbreiterung des QRS-Komplexes und eine Verlängerung des QTIntervalls an.

Angesichts der hohen Toxizität ist eine primäre Dekontamination bei hohen Dosen unbedingt indiziert, wenn sie rechtzeitig $(<1 \mathrm{~h})$ erfolgen kann. Dabei ist zu beachten, dass die Magenspülung das Krampfpotential erhöht und die Gabe von Aktivkohle daher vorzuziehen ist. In Spätfällen bei potentiell schweren Intoxikationen durch Retardpräparate ist die orthograde Darmspülung die Methode der Wahl. Zur Behandlung kardiotoxischer Symptome wird Natriumbikarbonat als Antidot eingesetzt. Es ist indiziert bei QRS-Verbreiterung $>120 \mathrm{~ms}$, ventri- kulären Arrhythmien und volumenrefraktärer Hypotonie und wird bolusweise (50-100 mmol, ev. wiederholt) verabreicht (sog. «Blutalkalinisierung»). Es gibt Hinweise darauf, dass durch die Erhöhung des pH die Affinität der TCA zu den Natriumkanälen reduziert wird und durch den erhöhten Natriumgradienten gleichzeitig ein vermehrter Natriumeinstrom in die Zelle erfolgen kann.

\section{Literatur}

1 Smilkstein MJ, Burton BT, Keene W, et al. Acute respiratory illness linked to use of aerosol leather conditioner - Oregon. December 1992. MMWR Morb Mortal Wkly Rep 1993;41:965-7.

2 Okonek S, Reineke HJ, Fabricius W, Preussner K. Vergiftungen durch Leder-Imprägnierungsprays. Deutsch Med Wochenschr 1983;108:1863-7.

3 Jinn Y, Akizuki N, Ohkouchi M, Inase N, Ichioka M, Marumo F. Acute lung injury after inhalation of water-proofing spray while smoking a cigarette. Respiration 1998;65:486-8.

4 Williams N, Atkinson GW, Patchefsky AS. Polymer-fume fever: not so benign. J Occup Med 1974;16:519-22.

5 Baud FJ, Sabouraud A, Vicaut E, Taboulet P, Lang J, Bismuth C, Rouzioux JM, Scherrmann JM. Brief report: treatment of severe colchicine overdose with colchicine-specific Fab fragments. N Engl J Med 1995;332:642-5.

6 Rauber C, Guirguis M, Schnorf S, Lorent JP, MeierAbt PJ, Kupferschmidt H. Tox-Zentrum 2000. Zum Jahresbericht des Schweizerischen Toxikologischen Informationszentrums. Schweiz Ärztezeitung 2001;82(41):2184-7.

\section{Notfallnummer 145}

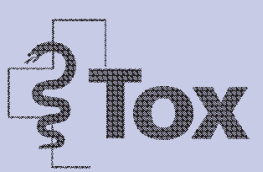

Ab dem 5. November 2003 kann das Tox über die nationale Notfallnummer 145 erreicht werden. Die bisherige Nummer 012515151 bleibt weiterhin in Betrieb. Unverändert bleibt auch die Nummer 012516666 für nicht dringliche Fälle. 